biosynthesis is central to biology; not only are structural proteins the building blocks for growth and enzymes the key to function, but also the specificity of structure of each protein and of the nucleic acids concerned in its synthesis is the basis of reproduction.

This is a most important book on this central theme. Dr. Medveder has not tried to cover the same ground as the numerous monographs and reviews on molecular biology, but has taken a fresh look at the problem and kept to the subject of this title. Throughout the author has avoided stressing historical or over-familiar aspects but has given critical accounts of the experimental work on which our present knowledge is based and of the theoretical implications of the results.

Some idea of the scope of this book is given by chapter headings: mechanism of activation of amino acids (III), selective transfer of activated amino acids (IV), the role of organelles (V), formation of antibodies (XI), morphogenetic changes-the genetic programme of development (XVIII), the problem of ageing at the molecular level (XIX). Particularly valuable is a supplementary chapter (XX) for the English edition dealing with important advances during 1963 and 1964 (the Russian edition appeared in 1962). Each chapter has a concluding section summing up the contents, and there is a very useful 'General Conclusion' at the end of each of the four parts into which the book is divided. A full bibliography appears at the end of each chapter.

The chapters are subdivided into sections, each with its own subheading, but the subject matter of each section and chapter leads so logically into the next that this arrangement helps the reader to go smoothly through from start to finish. This organization of the book is one of its most valuable features, contributing greatly to its readability and lucidity. Both author and translator are to be congratulated on this and on the clarity of the text; minor criticisms are the somewhat excessive use of inverted commas and the choice of 'matrix' instead of the more familiar 'template'. The absence of a subject index is unimportant in a book of this type; the logical arrangement of the text and the very complete list of contents make an index unnecessary.

This book should be required reading for every candidate for honours in biochemistry, genetics, or microbiology. Any student of biology or medicine who wished to understand modern biochemical genetics would be well advised to read it.

L. I. WoOLF

\section{Norrie's Disease. A Congenital Progressive} Oculo-acoustico-cerebral Degeneration. By Mette Warburg. Acta Ophthalmologica: Supplementum 89. (Pp. 147; illustrated. Dan. kr. 65.) Copenhagen: Ejnar Munksgaard. 1967.

This excellent monograph is based on an exhaustive study of 6 families showing congenital sex-linked pseudoglioma: 3 from Denmark and 3 from Sweden. They covered 35 cases, the youngest being 7 days old and the oldest 60 years. A detailed clinical analysis brings out that frequently there are associated mental retardation and impaired hearing, while the histological studies established malformations of the neuroepithelium of the retina, the optic tracts, and cerebral cortex. Inheritance was in a recessive sex-linked manner.

The recognition of this disorder has proceeded in a peculiarly halting manner for some 50 years. It is likely that the first case record goes back to Ernest Clarke in 1898. The pedigree of hereditary microphthalmia published by Ash and his associates in 1922, and subsequently elaborated by J. A. Fraser Roberts in 1937, by Iles in 1939, and by Whitnall and Norman in 1940, clearly established sex-linked congenital blindness associated with mental deficiency; these authors stressed the microphthalmia rather than the underlying 'pseudoglioma'. R. Wilson of London in recording another family in 1936 stressed the pseudoglioma aspect. Abroad, Heine, in 1925, invoked intrauterine inflammation to explain pseudoglioma in two brothers whose maternal uncle was blind, deaf, and mentally abnormal, while Norrie stressed, in 1927 and 1933, the occurrence of a congenital hereditary form of blindness in males in three families. It is possible that a brief account by Fernandez in 1905 on two brothers in Cuba is yet another early report of this affection. The disorder finally came to be fully recognized as a clinical entitysex-linked pseudoglioma-by W. M. G. Wilson in 1949 who published a particularly convincing Canadian pedigree of an unaffected woman who had two affected brothers and affected sons by three different men.

This monograph reviews fully the available literature which includes a number of recent reports. There is also a full discussion of allied conditions. Pseudoglioma and microphthalmos, the two aspects stressed in the earlier reports, are now regarded as secondary to nonattachment of the opposing layers of the invaginated optic cup. The designation of Norrie's disease was first applied by Andersen and Warburg in 1961, and more recent German literature speaks of Heine-Norrie disease, but it seems that any eponymous designation is questionable, particularly when the name sex-linked pseudoglioma is both more informative and less debatable.

ARNOLD SORSBY

Introduction to Animal Physiology and Physiological Genetics. By E. M. Pantelouris. International Series of Monographs in Pure and Applied Biology/Zoology Division/Volume 32. (Pp. viii +497; 172 figures +50 tables. 75s.) Oxford: Pergamon Press. 1967.

Medical practitioners and human geneticists will enjoy the author's broadly zoological approach to physiology. The book is arranged in four sections : response mechanisms, nutrition and energy-yielding processes, regulation 